\title{
Tracking Control of a Visual System
}

\author{
Masnori Sugisaka and Masayoshi Hara \\ Department of Electrical and Electronic Engineering, \\ Oita University, \\ Oita, Japan
}

\begin{abstract}
We developed a visual system. This system is able to track the moving objects within a certain range of errors. The visual system is driven by two DC motors. They are controlled based on the visual data obtained from a CCD video camera. The problem how to implement a fuzzy logic control method in this system is also considered in order to check the control performance of tracking. The fuzzy logic algorithm is a powerful control technique for nonlinear dynamical system.
\end{abstract}

\section{INTRODUCTION}

With the recent rapid development of computers, the practical control application and optimal control theories has progressed and its effectiveness is being demonstrated. Development of a system which is able to recognize and track the moving object, or recognize the shape and size of moving objects is necessary for constracting intelligent robots that assist humann beings.

Recentry, we developed a visual system. This system is able to track the moving objects within a certain range of errors. The visual system is driven by two DC servo motors with encoders. These two DC servo motors are controlled by a computer based on the visual data obtained from a CCD video camera. The software to track the moving objects is developed based on the pulse wide modulation (PWM) of the DC motors. The duty ratio of PWM is decided from the experiments considering the trackspeed of the visual system. In the control study, conventional proportional control is used at first in order to get the data concerning the fundamental movement of the system designed. Also, the problem how to implement a fuzzy logic control method in this system is also considered in order to check the control performance of tracking. The fuzzy logic algorithm is a powerful control technique for nonlinear dynamical system.

This paper explains briefly the method and results obtained in the experiments. The configuration of the tracking system is introduced in Section 2. Section 3 explains the processing of the image data from the CCD camera for tracking briefly. In Section 4 the tracking controls methods are discussed. Finally, conclusion follows in Section 5. 


\section{THE CONFIGURATION OF VISUAL SYSTEM}

The configuration of tracking system developed in our laboratory is shown in Fig.l. As shown in Fig.l the system consists of two DC motors with two encorders, a CCD video camera. One DC moter with encoder which moves in up and down direction is located in the upper sphere. The othe DC moter with encoder which moves in right and left directions is located in low box. The horizontal and vertical DC moters are driven by the PWM controller of electric circuits and the velocity of tracking is selected appropriately by considering both the hardware limitations and the velocity of an objects to be tracked. Namely, the duty ratio of the amerture current is changed.

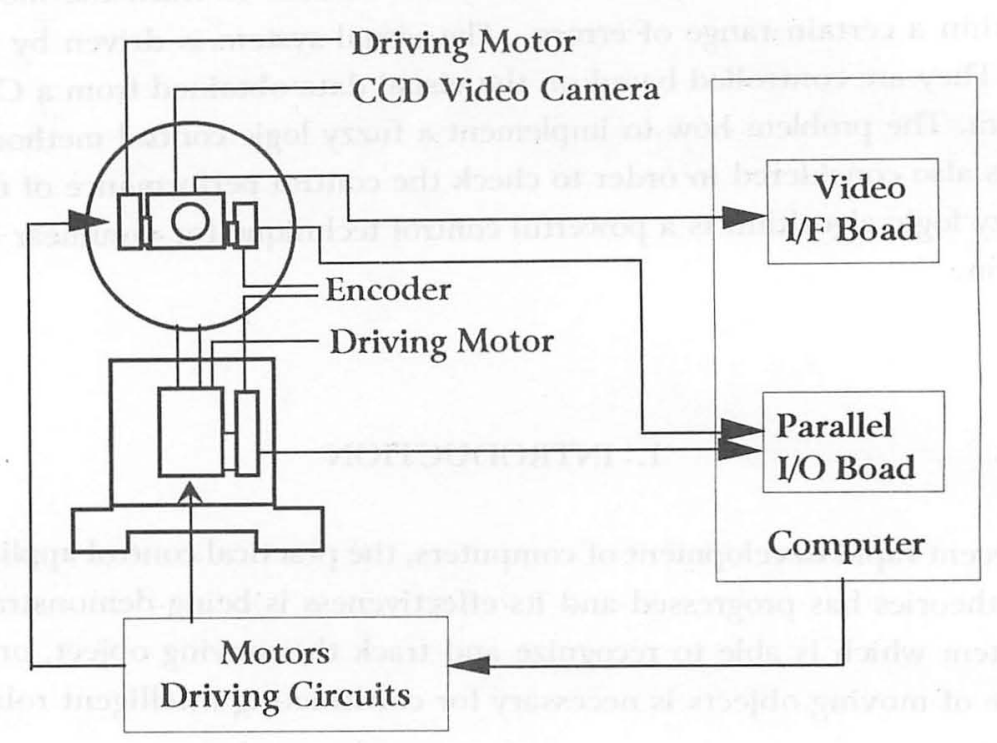

Fig. 1 The configuration of visual system

The CCD video camera is able to move 60 degree in right and left directions from the center and is able to move 45 degree in up and down directions from the center. The movement of DC motors is locked from hardware in the outside of the region stated above even if the PWM controller received on-signal from the microcomputer. The hexadecimal values from $00 \mathrm{~h}$ to $\mathrm{FFh}$ are assigned for the values from 0 degree to 360 degree in the revolutional angles of the visual system along the clockwise direction in horizontal and vertical directions where the center of the visual system is assumed to be located at $00 \mathrm{~h}$. Therefore the movable angle is equal to 360 degree/256 bits and 1.40625 degrees correspond to $1 \mathrm{H}$. In decimal digit the visual system is able to move from \pm 60 degrees $/ 1.40625$ degrees $= \pm 42.667$ in right and left directions of horizontal axis, respectively and is able to move from \pm 45 degrees $/ 1.40625$ degrees $= \pm 32$ in up and down directions of vertical axis, respectively. 
The image data of a picture with $16 \times 12$ (low $\times$ column) $=192$ pixels are taken by the CCD video camera at one sampling and has values of six bits, namely, 64 degrees of intensity. The value of the image data is expressed as hexadecimal digit.

The image data is taken into the NEC PC980l microcomputer through the video interface and is stored in the memory. Thereafter, the image data is read from the memory and caluculated. This processing time is approximately less thean $30 \mathrm{~ms}$ and therfore the sampling period is chosen $30 \mathrm{~ms}$ in the experiments.

\section{PROCESSING OF IMAGE DATA FROM CCD CAMERA}

For the purpose of tracking, the highest degree of intensity is selected. The camera is moved in order for the current position to became the center of the image. In oter words, the deviation between the position $(\mathrm{i}, \mathrm{j})$ stated above and the center of the image is calculated. Thereafter the position of camera to be moved is calculated and the camera is moved to this position.

As to tracking of visual system, the following procedures are repeated:

1. The image data of picture of a moving objects is taken into the microcomputer from the CCD camera.

2. The position $(i, j)$ of the highest degree of intensity of the CCD video camera is detected.

3. The position $(i, j)$ of the highest degree of intensity of the image is detected.

4. The current position of the CCD video camera is read and taken into the microcomputer.

5. The deviation between the position $(i, j)$ stated above and the center of the image is calculated.

6. The camera is moved to this position.

The above procedures are performed repeatedly in order to track the moving objects in a certain range of errors. The software to track the moving objects is developed based on the PWM of the DC motors. The duty ratio employed is decided from the experiments considering the trackspeed of the visual system.

\section{CONTROL METHODS}

In the control study, conventional proportional control is used at first in order to get the data concerning the fundamental movement of the system designed. Also, the problem how to implement afuzzy logic control method in this system is also considered in order to check the control performance of tracking.

The fuzzy-logic control rule is described by if $\sim$ then form. The menbership function of vertical and horizontal direction are shown Fig. 2 and Fig. 3. The rules table which is used in the experiments is shown Table 1. 


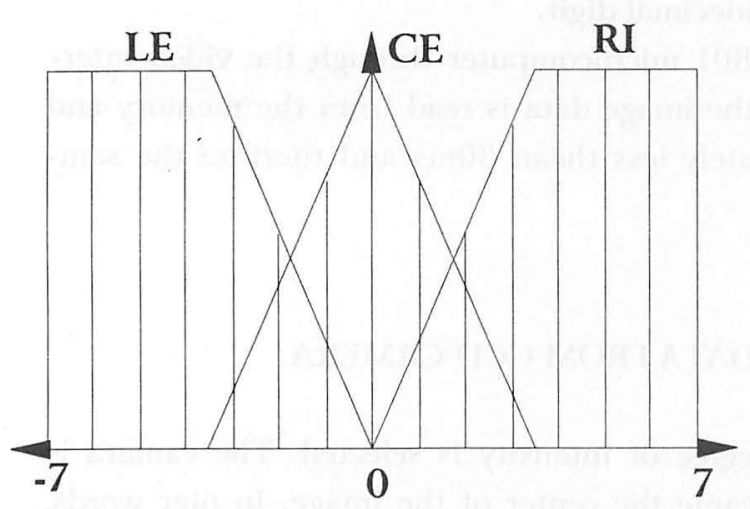

Fig. 2(a) Menbership function of e

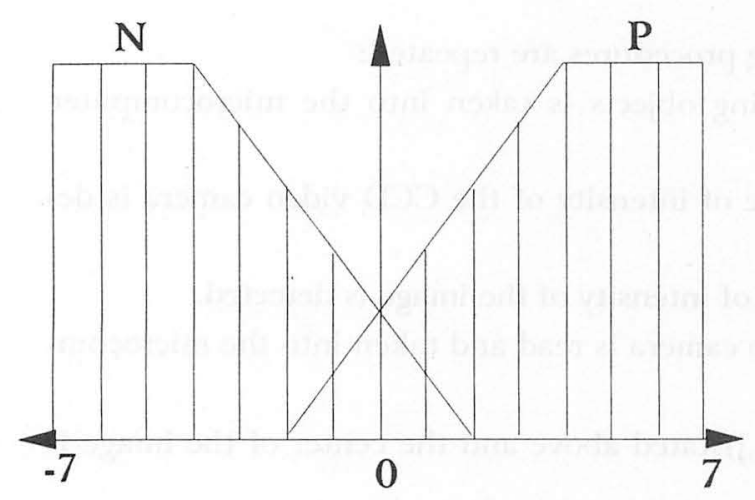

Fig. 2(b) Menbership function of $\Delta \mathrm{e}$

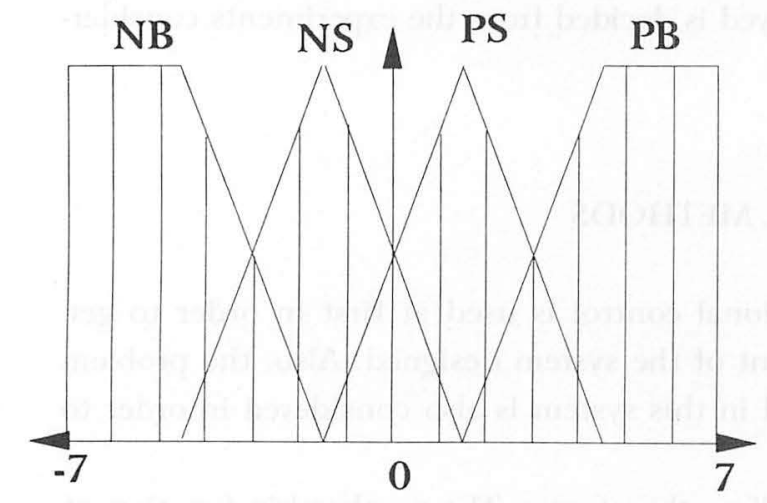

Fig. 2(c) Menbership function of $u$

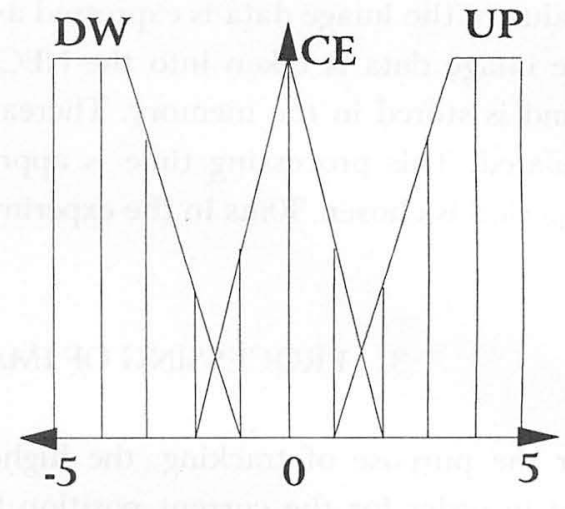

Fig. 3(a) Menbership function of e

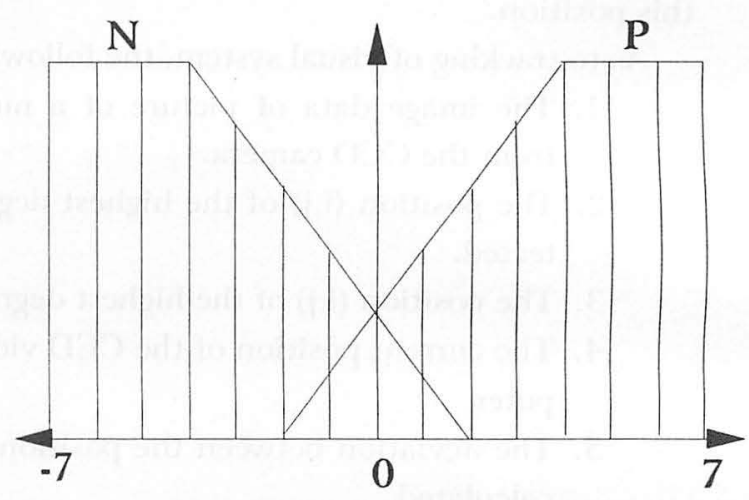

Fig. 3(b) Menbership function of $\Delta \mathrm{e}$

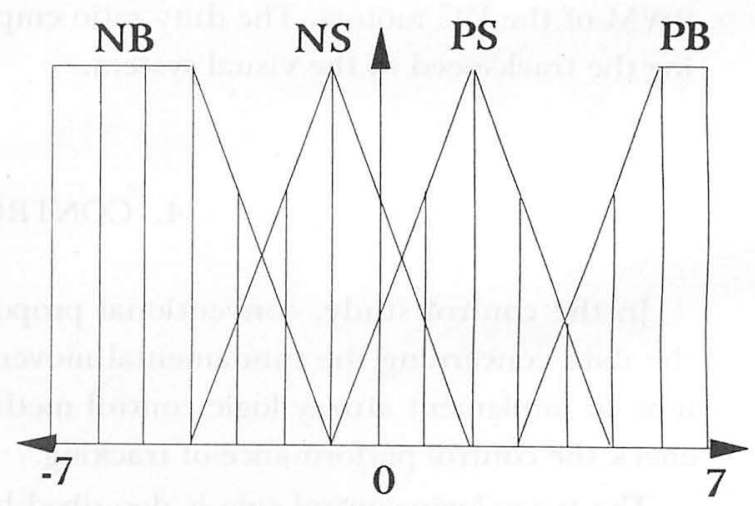

Fig. 3(c) Menbership function of $u$ 
where

\begin{tabular}{|c||c|c|c|}
\hline$\Delta \mathrm{e}^{\mathrm{e}}$ & $\mathrm{RI}$ & $\mathrm{CE}$ & $\mathrm{LE}$ \\
\hline \hline $\mathrm{P}$ & $\mathrm{PB}$ & $\mathrm{PS}$ & $\mathrm{ZO}$ \\
\hline $\mathrm{N}$ & $\mathrm{ZO}$ & $\mathrm{NS}$ & $\mathrm{NB}$ \\
\hline
\end{tabular}

Table 1 Fuzzy rules table

$$
\begin{aligned}
& e_{n}=y_{n}-r_{n}, \\
& \Delta e_{n}=e_{n}-e_{n-1},
\end{aligned}
$$

$y_{n}:$ the position of visual system

$r_{n}$ : the position of a moving object

$\mathrm{e}_{\mathrm{n}}$ : the deviation

$\Delta e_{n}:$ is the difference of deviation

The label of fuzzy variables $e_{n}$ is right(RI), center(CE), and left(LE). upper(UP), down(DW)

The label of fuzzy variables $\Delta e_{n}$ is positive $(P)$ and negative(N).

The fuzzy reasoning used is min-max method.

The experiment result of tracking control using fuzzy control is shown Fig.4.

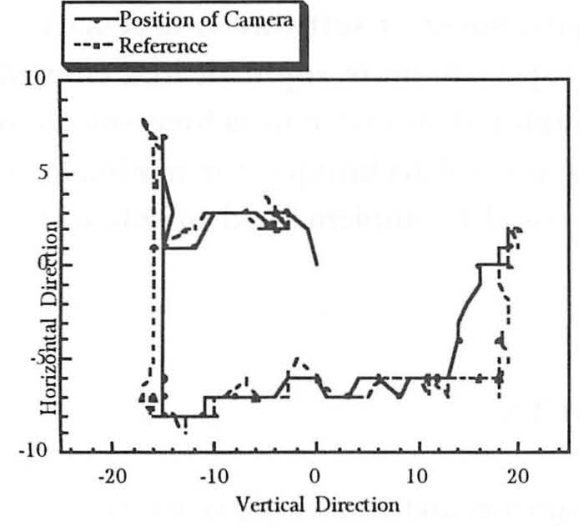

Fig. 4(a) Experiment result of fuzzy control

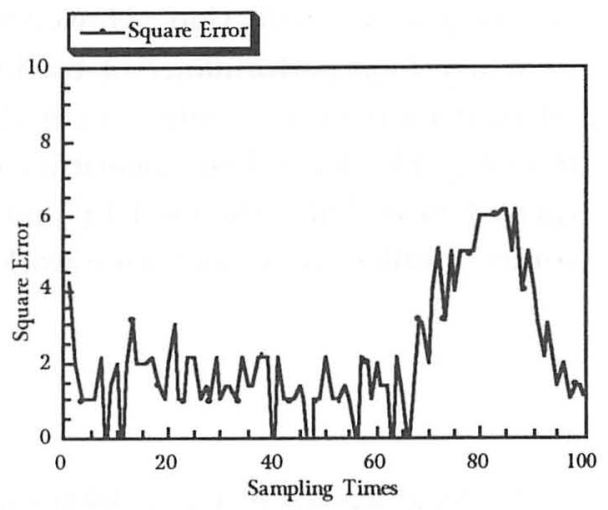

Fig. 4(b) Error 


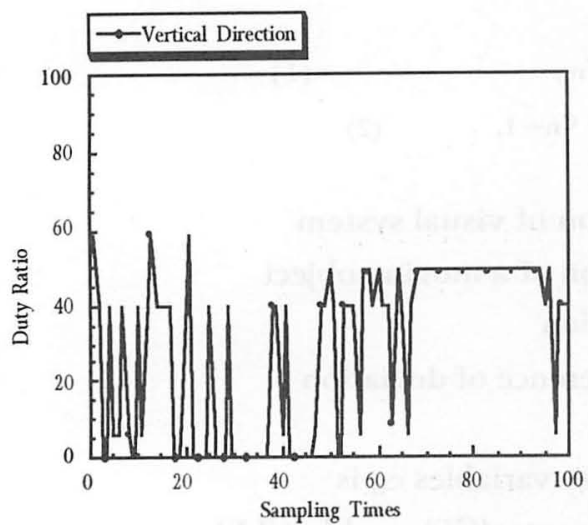

Fig. 4(c) Duty Ratio of Vertical Direction

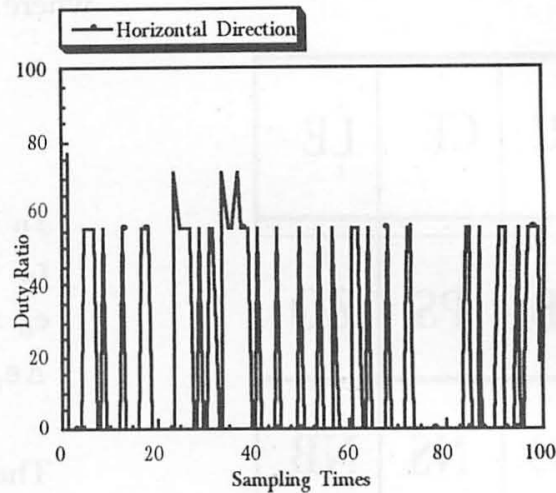

Fig. 4(d) Duty Ratio of Vertical Direction

\section{CONCLUSION}

In this paper the tracking system of moving objects developed in our laboratory is introduced and is explained briefly. The method how to track a moving objects is also shown. It is seen from the fundmentalexperiments performed that the system developed is able to track the moving objects of slow speed quite satisfactory at this stage and works at a certain degree of satisfaction. However farthes improvement of software is necessary in order to achive high performance of tracking control. Especialy more sophisticated control method than propotional control method could be employed in order to achive smoth and fast tracking. The fuzzy logic algorithm is a powerful control techinique for nonlinear dynamical system and also the model predictive control could be implemented in this system. The control studies are in our future works.

\section{REFERENCES}

[1] M. Sugisaka and H. Kaita, PWM Control of Recognition and Tracking System for Moving objects, The 3Ith SICE Annual Conference, , Kumamoto, 1992.

[2] M. Sugisaka and M. Eto, Fuzzy Contr ol of Mobile Vehicle, IFAC Workshop on Automatic Control for Quality and Productivity, ACQP '92, Preprints, Vol.2, Editors A. Kuzucu, I. Eksin and A. T. dinibutun, pp.687-694, Istanbul, Turkiya, 1992.

[3] M. Sugisaka and M. Ino, Model Predictive Contr ol based on Neur al Identification Method, 5th Internatinal Workshop of the Bellman Continuum, Honolulu, Hawai, USA, 1993. 